\title{
Local finiteness, distinguishing numbers, and Tucker's conjecture
}

\author{
Florian Lehner* \\ Department of Mathematics \\ University of Hamburg \\ Hamburg, Germany \\ mail@florian-lehner.net
}

\author{
Rögnvaldur G. Möller \\ School of Engineering and Natural Sciences \\ University of Iceland \\ Reykjavik, Iceland \\ roggi@hi.is
}

Submitted: Dec 4, 2014; Accepted: Oct 15, 2015; Published: Oct 30, 2015

Mathematics Subject Classifications: 05C25, 05C63, 05C15

\begin{abstract}
A distinguishing colouring of a graph is a colouring of the vertex set such that no non-trivial automorphism preserves the colouring. Tucker conjectured that if every non-trivial automorphism of a locally finite graph moves infinitely many vertices, then there is a distinguishing 2-colouring.

We show that the requirement of local finiteness is necessary by giving a nonlocally finite graph for which no finite number of colours suffices.
\end{abstract}

\section{Introduction}

A colouring of the vertices of a graph $G$ is called distinguishing if no non-trivial automorphism of $G$ preserves the colouring. This notion was first studied by Albertson and Collins [1, motivated by a recreational mathematics problem posed Rubin [9].

While a distinguishing colouring clearly exists for every graph (simply colour every vertex with a different colour), finding a distinguishing colouring with the minimum number of colours can be challenging.

For infinite graphs one of the most intriguing questions is whether or not the following conjecture of Tucker [12] is true.

Conjecture 1. Let $G$ be an infinite, connected, locally finite graph with infinite motion. Then there is a distinguishing 2-colouring of $G$.

This conjecture can be viewed as a generalisation of a result on finite graphs due to Russell and Sundaram [10]. It is known to be true for many classes of infinite graphs

*The author acknowledges the support of the Austrian Science Fund (FWF), project W1230-N13. 
including trees [13], tree-like graphs [4], and graphs with countable automorphism group [5]. In [11] it is shown that graphs satisfying the so-called distinct spheres condition have infinite motion as well as distinguishing number two. Examples for such graphs include leafless trees, graphs with infinite diameter and primitive automorphism group, vertex-transitive graphs of connectivity 1, and Cartesian products of graphs where at least two factors have infinite diameter. It is also known that Conjecture 1 is true for graphs fulfilling certain growth conditions [7]. In [8] it is shown that for locally finite graphs random colourings have a good chance of being distinguishing.

Many of the above results also hold for non-locally finite graphs which raises the question, whether the condition of local finiteness in Tucker's conjecture can be dropped.

A first indication, that local finiteness may be necessary has been given in the setting of permutation groups acting on countable sets. Here, instead of considering the automorphism group of a graph acting on the vertex set, we consider (faithful) group actions. A generalization of Conjecture 1 to this setting has been given by Imrich et al. [5].

Conjecture 2. Let $\Gamma$ be a closed, subdegree finite permutation group on a set $S$. Then there is a distinguishing 2-colouring of $S$.

For this generalization subdegree finiteness (which plays the role of local finiteness) is known to be necessary [6].

In this short note we show that local finiteness is also necessary in the graph case. More precisely we give a non-locally finite, arc transitive graph with infinite motion which does not admit a distinguishing colouring with any finite number of colours.

\section{Preliminaries}

Throughout this paper we will use Greek letters for group related variables while the Latin alphabet will be reserved for sets on which the group acts.

Let $S$ be a countable set and let $\Gamma$ be a group acting faithfully (i.e. the identity is the only group element which acts trivially) on $S$ from the left. The image of a point $s \in S$ under an element $\gamma \in \Gamma$ is denoted by $\gamma s$.

The stabilizer of $s$ in $\Gamma$ is defined as the subgroup $\Gamma_{s}=\{\gamma \in \Gamma \mid \gamma s=s\}$. We say that $\Gamma$ is subdegree finite if for every $s \in S$ all orbits of $\Gamma_{s}$ are finite.

The motion of an element $\gamma \in \Gamma$ is the number (possibly infinite) of elements of $S$ which are not fixed by $\gamma$. The motion of the group $\Gamma$ is the minimal motion of a non-trivial element of $\Gamma$. Notice that the motion is not necessarily finite, in fact all groups considered in this paper have infinite motion. The motion of a graph $G$ is the motion of Aut $G$ acting on the vertex set.

Let $C$ be a (usually finite) set. A $C$-colouring of $S$ is a map $c: S \rightarrow C$. Given a colouring $c$ and $\gamma \in \Gamma$ we say that $\gamma$ preserves $c$ if $c(\gamma s)=c(s)$ for every $s \in S$. Call a colouring distinguishing if no non-trivial group element preserves the colouring. 


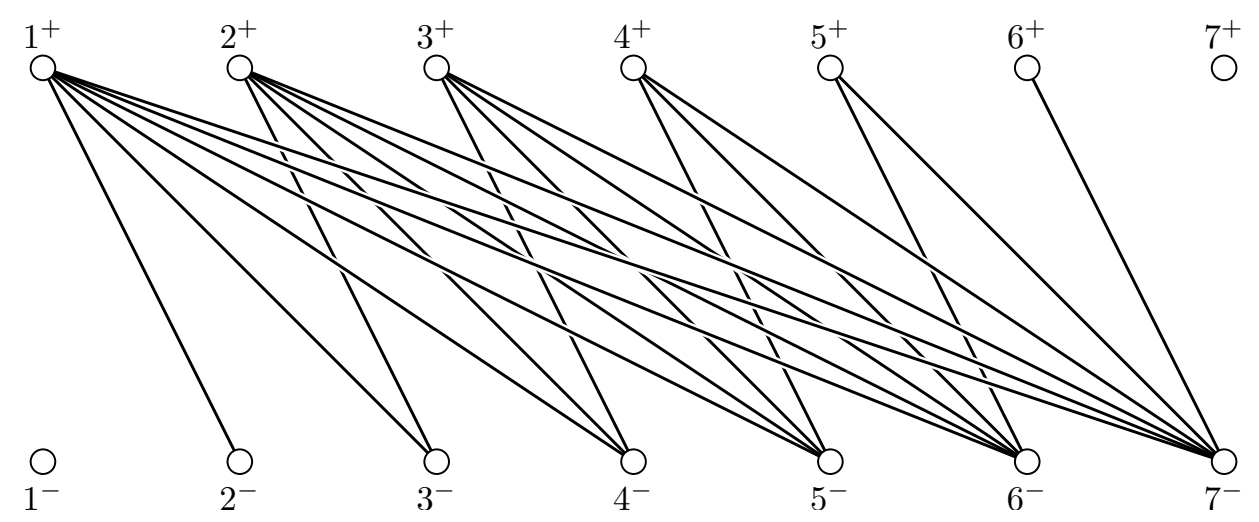

Figure 1: An induced subgraph of the graph in Theorem 4. Note that edges only go from top left to bottom right. By the definition of the graph all such edges are present and every edge is of this type.

\section{The example}

The construction that we use relies on the following result from [6] which also shows that there are permutation groups on a countable sets whose distinguishing number is infinite. The proof uses a standard back-and-forth argument, see for example [2, Sections 9.1 and 9.2 ] and [3, Sections 2.8 and 5.2].

Theorem 3 (Laflamme et al. [6]). Let $\Gamma$ be the group of order automorphisms of $\mathbb{Q}$ (i.e. bijective, order preserving functions $\gamma: \mathbb{Q} \rightarrow \mathbb{Q})$. Then $\Gamma$ has infinite motion but no distinguishing colouring with finitely many colours.

Clearly the group $\Gamma$ of the above theorem is the full automorphism group of the following directed graph: take $\mathbb{Q}$ as vertex set and draw an edge from $q$ to $r$ if $q \leqslant r$. The underlying undirected graph is the complete countable graph which also has infinite distinguishing number but finite motion.

Theorem 4. There is a countable, connected, arc transitive graph with infinite motion which has no distinguishing colouring with a finite number of colours.

Proof. Let $\mathbb{Q}^{+}$and $\mathbb{Q}^{-}$be two disjoint copies of $\mathbb{Q}$. Denote the elements corresponding to $q \in \mathbb{Q}$ in these copies by $q^{+}$and $q^{-}$, respectively. Consider the (undirected) graph $G=(V, E)$ where $V=\mathbb{Q}^{+} \cup \mathbb{Q}^{-}$and $q^{+} r^{-} \in E$ whenever $q<r$. Figure 1 shows a small subgraph of this graph to give an idea of what it looks like. Clearly $G$ is countable and connected.

Note that $G$ is bipartite with bipartition $\mathbb{Q}^{+} \cup \mathbb{Q}^{-}$. Hence every automorphism $\gamma$ of $G$ either fixes $\mathbb{Q}^{+}$and $\mathbb{Q}^{-}$set-wise, or swaps the two sets. Furthermore if $\gamma q^{+}=r^{+}$then $\gamma q^{-}=r^{-}$because $q^{-}$is the unique vertex with the property $N\left(q^{-}\right)=\bigcap_{v \sim q^{+}} N(v) \backslash\left\{q^{+}\right\}$. A similar argument shows that if $\gamma q^{+}=r^{-}$then $\gamma q^{-}=r^{+}$. So the action on $\mathbb{Q}^{+}$uniquely determines an automorphism of $G$. 
Now, we define a family of automorphisms of $G$ (we will later show that these are in fact all the automorphisms of $G$ ). For every order automorphism $\gamma$ of $\mathbb{Q}$, define the functions $\gamma_{\uparrow}$ and $\gamma_{\downarrow}$ as follows:

- $\gamma_{\uparrow}$ applies $\gamma$ to both copies of $\mathbb{Q}$, i.e. $\gamma_{\uparrow}\left(q^{+}\right)=(\gamma(q))^{+}, \gamma_{\uparrow}\left(q^{-}\right)=(\gamma(q))^{-}$,

- $\gamma_{\downarrow}$ first applies $\gamma$ to both copies, then reverses the order on each of them and swaps them, i.e. $\gamma_{\downarrow}\left(q^{+}\right)=(-\gamma(q))^{-}$, and $\gamma_{\downarrow}\left(q^{-}\right)=(-\gamma(q))^{+}$.

It is straightforward to check that these maps are indeed automorphisms of the graph $G$.

To see that $G$ is arc transitive, notice that the arc $0^{+} 1^{-}$can be mapped to any arc of the form $q^{+} r^{-}$by the automorphism $\gamma_{\uparrow}$ where

$$
\gamma(x)=q+(r-q) x
$$

The map $\gamma$ is an order automorphism of $\mathbb{Q}$ since $q^{+} r^{-} \in E$ implies that $q<r$. By analogous arguments, the arc $0^{+} 1^{-}$can be mapped to any arc of the form $q^{-} r^{+}$by the automorphism $\gamma_{\downarrow}$ where

$$
\gamma(x)=-q+(q-r) x .
$$

Every map of the type $\gamma_{\uparrow}$ and $\gamma_{\downarrow}$ moves infinitely many vertices. Thus, to show that $G$ has infinite motion, it suffices to prove that the automorphisms of the form $\gamma_{\uparrow}$ and $\gamma_{\downarrow}$ as defined above are the only automorphisms of $G$.

It is not hard to see that $q \geqslant r$ if and only if $N\left(q^{+}\right) \subseteq N\left(r^{+}\right)$. This implies that $N\left(\phi\left(q^{+}\right)\right) \subseteq N\left(\phi\left(r^{+}\right)\right)$for every automorphism $\varphi$ of $G$. If $\varphi$ fixes $\mathbb{Q}^{+}$set-wise we conclude that $\varphi$ preserves the order on $\mathbb{Q}^{+}$, hence it is equal to $\gamma_{\uparrow}$ for a suitable order automorphism $\gamma$. An analogous argument shows that if $\varphi$ swaps $\mathbb{Q}^{+}$and $\mathbb{Q}^{-}$, then $\varphi=\gamma_{\downarrow}$ for an order automorphism $\gamma$ of $\mathbb{Q}$.

Finally, assume that there is a distinguishing colouring $c$ of $G$ with $n<\infty$ colours. In particular this colouring would break every automorphism of the form $\gamma_{\uparrow}$. Hence the map $q \mapsto\left(c\left(q^{+}\right), c\left(q^{-}\right)\right)$would be a distinguishing colouring of $\mathbb{Q}$ with $n^{2}<\infty$ colours, a contradiction to Theorem 3 .

\section{References}

[1] M. O. Albertson and K. L. Collins. Symmetry breaking in graphs. Electron. J. Combin., 3(1):\#R18, 1996.

[2] M. Bhattacharjee, D. Macpherson, R. G. Möller, and P. M. Neumann. Notes on infinite permutation groups. Springer Lecture Notes Series 1698. Springer, 1998.

[3] P. J. Cameron. Oligomorphic permutation groups. LMS Lecture Notes Series 152. Cambridge: Cambridge University Press, 1990.

[4] W. Imrich, S. Klavžar, and V. Trofimov. Distinguishing infinite graphs. Electron. J. Combin., 14(1):\#R36, 2007. 
[5] W. Imrich, S. M. Smith, T. Tucker, and M. E. Watkins. Infinite motion and 2distinguishability of graphs and groups. J. Algebr. Comb., 41(1):109 - 122, 2015.

[6] C. Laflamme, L. Nguyen Van Thé, and N. Sauer. Distinguishing number of countable homogeneous relational structures. Electron. J. Combin., 17(1):\#R20, 2010.

[7] F. Lehner. Distinguishing graphs with intermediate growth. Combinatorica, to appear.

[8] F. Lehner. Random colorings and automorphism breaking in locally finite graphs. Combin. Probab. Comput., 22(6):885 - 909, 2013.

[9] F. Rubin. Problem 729. J. Recreational Math., 11:128, 1979. (solution in volume 12, 1980).

[10] A. Russell and R. Sundaram. A note on the asymptotics and computational complexity of graph distinguishability. Electron. J. Combin., 5:\#R23, 1998.

[11] S. M. Smith, T. W. Tucker, and M. E. Watkins. Distinguishability of infinite groups and graphs. Electron. J. Combin., 19(2):\#R27, 2012.

[12] T. W. Tucker. Distinguishing maps. Electron. J. Combin., 18(1):\#P50, 2011.

[13] M. E. Watkins and X. Zhou. Distinguishability of locally finite trees. Electron. J. Combin., 14(1):\#R29, 2007. 